\title{
Comparación de angiografía coronaria con el ultrasonido intracoronario como patrón de referencia en el diagnóstico de vasculopatía post trasplante cardíaco.
}

Humberto Torres ${ }^{1,3}$, Mauricio Aninat $t^{1,3}$, Luis Becerra ${ }^{1,3}$, Lorenzo Merello ${ }^{1,3}$, Sergio Ramos ${ }^{2,3 a}$, Alex Mora Matra $^{2,3 a}$,
Patricia Valenzuela

Unidad de Hemodinamía Hospital Gustavo Fricke Viña del Mar

1 Universidad de Valparaíso, Facultad de Medicina, Escuela de Medicina,

Valparaíso, Chile.

2 Universidad, Andrés Bello, Facultad de Medicina, Escuela de Tecnología

Médica. Chile.

3 Hospital Dr. Gustavo Fricke Viña del Mar, Chile.

a Tecnólogo Médico, b Enfermera, c Estadístico, d Alumnos tesistas

\section{Resumen:}

Antecedentes: La Vasculopatía del injerto (VDI) es la principal causa de muerte tardía del trasplante cardiaco (TX). Un diagnóstico precoz de esta complicación tendría un impacto en la terapia y pronóstico de esta afección. El ultrasonido intracoronario (IVUS), permite un diagnóstico precoz y certero de VDI, pero en la mayoría de los centros aún se utiliza la coronariografía.

Objetivo: Evaluar la validez de la coronariografía en el diagnostico de VDI en pacientes trasplantados cardiacos, comparado con el IVUS.

Metodología: Estudio transversal, en el cual se analizó una muestra de 36 pacientes con un tiempo promedio de trasplante de $3,7 \pm 3,7$ años, a quienes se realizó simultáneamente un examen de coronariografía y un IVUS. Se evaluó la reproducibilidad contrastando los resultados de la interpretación visual de la coronariografía versus la coronariografía con análisis cuantitativo. Se calculó la Sensibilidad, Especificidad, Valores predictivos (VPP, VPN), Likelihoods y el coeficiente de equivalencia de Spearman-Brown. Resultados: La coronariografía cualitativa mostró ser más exacta que la cuantitativa, con una sensibilidad 30,4\% [95\% IC $=11,6-49,2]$, una especificidad $92,3 \%$ [95\% IC $=77,8-106,7]$, VPP $87,5 \%$ $[95 \%$ IC $=64,5-110,4], \mathrm{VPN} 42,8 \% \quad[95 \%$ IC $=24,5$ $-61,1], \mathrm{LR}(+) 3,9[95 \%$ IC $0,55-28,7]$ y un LR (-) $0,75$ [95\% IC $=0,55-1,03]$. La coronariografía cualitativa y cuantitativa son moderadamente equivalentes con un coeficiente de equivalencia Spearman Brown de 0,65 .

Conclusión: La validez y la reproducibilidad de la coronariografía en el paciente con TX es moderada y debería ser complementada con IVUS para el diagnóstico de VDI.

\section{Correspondencia:}




\section{Comparison of coronary angiography and intra-coronary ultrasound as "gold standard" in the diagnosis of post cardiac transplantation vasculopathy}

Background: Graft vasculopathy (GV) is the main cause of late death following cardiac transplantation (TX). Early diagnosis of this condition may have an impact upon treatment and prognosis of this complication. Intravascular ultrasound (IVUS) allows an early and accurate diagnosis of GV. However, most centers continue to use coronary angiography for this purpose. Aim: to evaluate coronary angiography for the diagnosis of GV in relation to IVUS in post TX patients.

Methods: In a cross-sectional study the results of coronary angiography and IVUS, used as gold standard, were compared in 36 patients with a mean post TX follow up of $3.7 \pm 3.7$ years. Results were compared between visual and quantitative coronary angiography. Sensitivity, specificity, positive and negative predictive values (PPV, NPV), likelihood ratios and the equivalence Spearman-Brown coefficient were calculated.

\section{Introducción}

La Vasculopatía del Injerto (VDI) es la primera causa de muerte en la evolución alejada del trasplante cardíaco (TX) ${ }^{1}$. Su etiología es multifactorial; hay factores inmunológicos y no inmunológicos que tienen influencia diferente en cada paciente ${ }^{2}$. Dentro de estos últimos también se han invocado factores étnicos y es así que en una población de trasplantados cardíacos chinos se describe una incidencia de $2 \%$ de VDI, evaluados por coronariografía a los 4 años de evolución post trasplante ${ }^{3}$. Si bien la incidencia es variable de acuerdo a las características de la población, en general se acepta que un $50 \%$ de los pacientes trasplantados desarrolla VDI a los 5 años post trasplante ${ }^{4}$. El inicio puede ser muy precoz y su evolución progresiva dependiendo de los factores causales y su potencial tratamiento. La coronariografía es el método diagnóstico clásico en conjunto con la evaluación de la funcionalidad del injerto que tiene la limitante de ser poco sensible y tardío. En el último tiempo se ha desarrollado el IVUS, el cual, es un método de diagnóstico más precoz pues permite una imagen directa de la pared vascular y de-
Results: Visual evaluation of coronary angiography was more accurate than quantitative coronary angiography. The sensitivity for $\mathrm{GV}$ was $30.4 \%$ (95\% C.I. 11.6 - 49.2), specificity $92.3 \%$ (95\% C.I. $77.8-106.7$ ), PPV 87.5\% (95\% C.I. 64.5 - 110.4, NPP 42.8\% (95\% C.I. 24.5 - 61.1), likelihood ratio (+) 3.9 (95\% C.I. 0.55 - 28.7), likelihood ratio (-) 0.75 (95\% C.I. 0.55 1.03). The Spearman Brown coefficient between visual and quantitative coronary angiography evaluation was 0.65 .

Conclusion: Accuracy and reproducibility of coronary angiography in the evaluation of GV is limited. IVUS should be used for better identification of GV.

Key words: Cardiac transplantation, Graft vasculopathy, Coronary angiography, Intracoronary ultrasound.

tecta los cambios en el engrosamiento intimal desde las 6 semanas post TX, convirtiéndolo en un poderoso marcador pronóstico de la VDI ${ }^{5}$. Esto permitiría un tratamiento más oportuno de los factores etiológicos así como también ajustar el esquema inmunosupresor con drogas que tengan un efecto más específico sobre la vasculopatía. El control de esta complicación puede prolongar el tiempo del injerto y la calidad de vida del paciente trasplantado ${ }^{6}$.

De allí la importancia de conocer la magnitud real de la capacidad diagnóstica de la coronariografía por sí sola, para detectar VDI, contrastándola con el método considerado actualmente como el más precoz y específico como es el IVUS.

\section{Metodología}

El estudio se llevó a cabo en la Unidad de Hemodinamía del Hospital Dr. Gustavo Fricke de Viña del Mar, el cual es uno de los dos centros actuales de referencia nacional para trasplante cardíaco.

Tipo de Estudio: Corresponde a un estudio de evaluación de pruebas diagnósticas de un diseño transversal 
con reclutamiento prospectivo.

Muestra: Estuvo constituida por pacientes trasplantados cardíacos del Hospital Dr. Gustavo Fricke, entre 18 a 58 años, controlados entre marzo 2008 - septiembre 2010, a quienes como parte de su protocolo de control se les realizó simultáneamente un examen de coronariografía y un estudio de IVUS, previo consentimiento informado institucional para participar en esta investigación. Se analizaron 36 pacientes con un tiempo promedio de trasplante de 3,7 $\pm 3,7$ años.

Criterios de Exclusión: Pacientes sin coronariografía previa. Pacientes que presenten contraindicación para realizarse el examen debido a malos accesos vasculares que imposibiliten la realización del procedimiento, insuficiencia renal descompensada (Creatinina sobre $3.0 \mathrm{mg} / \mathrm{dl}$ al momento del examen y en ascenso) y pacientes que al momento del examen se encuentren realizando un episodio de rechazo agudo.

\section{Variables a analizar}

VDI según coronariografía cualitativa: Se entiende por cualitativa a la interpretación visual de la coronariografía tomando en cuenta una disminución difusa o segmentaria del lumen coronario en relación a la coronariografía previa.

VDI según coronariografía cuantitativa: se entiende por variable cuantitativa al valor del diámetro de la estenosis, expresado en porcentaje, que diagnóstica VDI tomando en cuenta los parámetros consensuados por la Sociedad Internacional de Trasplante de Corazón y Pulmón (SITCP) el año $2010^{8}$.

VDI según grosor intimal: Medida otorgada por el equipo IVUS, expresada en mm que refleja el engrosamiento de la íntima. Sobre $0,5 \mathrm{~mm}$ de grosor intimal se considera vasculopatía post trasplante ${ }^{8}$.

\section{Procedimientos diagnósticos:}

Coronariografía: Se realizó la coronariografía según el protocolo de rutina.

\section{Mediciones post procedimiento de la coronariogra-} fía.

Se revisó todo el árbol coronario buscando lesiones estenóticas las cuales son medidas con el software QCA del Angiógrafo Siemens "Axiom Artis dfC versión VB11".

\section{Puntos de Corte de la coronariografía:}

Para determinar el punto de corte de VDI según la coronariografía se utilizará los criterios consensuados de la SITCP, del año 2010. Ver tabla $\mathrm{N}^{\circ} 1$.

\begin{tabular}{|c|c|}
\hline Código $]$ & Criterio \\
\hline VDIO & $\begin{array}{l}\text { (No[signif cativa)[ho[hay/lesiones[detectables[] } \\
\text { angiográf camente. }\end{array}$ \\
\hline $\begin{array}{l}\text { VDII } \\
0 \\
0 \\
0\end{array}$ &  \\
\hline VDI2] & 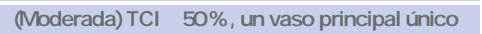 \\
\hline $\begin{array}{l}0 \\
0 \\
\square\end{array}$ &  \\
\hline $\begin{array}{l}0 \\
\text { VDI3 } \\
0 \\
0 \\
0 \\
0 \\
0 \\
0\end{array}$ &  \\
\hline
\end{tabular}

Donde:

\section{VDI0 = Ausencia de VDI \\ VDI1, VDI2, VDI3 = Presencia de VDI}

IVUS: Inmediatamente después de la coronariografía, se llevó a cabo un estudio con IVUS utilizando un catéter guía coronario $6 \mathrm{~F}$. A través este se introdujo una guía de 0,014 " hasta la porción distal de la arteria descendente anterior, se avanzó sobre la guía un catéter de ultrasonido Eagle Eye de $20 \mathrm{MHz}$, conectado a la consola del equipo Volcano s5TM Imaging. Finalmente, se procedió a realizar el retiro automático a una velocidad de $0,5 \mathrm{~mm} / \mathrm{seg}$, visualizando todo el tercio medio de la arteria.

\section{Mediciones post procedimiento del IVUS}

Se lleva a cabo la medición del área del lumen (LA) y del área total compuesta por el lumen más la íntima (TA). A su vez se realizó la medición de los diámetros mayores (DM) y menores (Dm) tanto del TA y el LA. De esta forma, mediante la siguiente fórmula podemos obtener un promedio del grosor intimal en el frame:

Grosor Intimal $(m m)=\underline{(D M T A-D M L A)+(D m T A-D m L A)}$ 2

\section{Puntos de Corte del IVUS:}

Ausencia de VDI $=$ Grosor intimal $<0,5 \mathrm{~mm}$.

Presencia de VDI= Grosor intimal $>0,5 \mathrm{~mm}$. 


\section{Análisis Estadístico}

\section{Exactitud diagnóstica:}

Tablas de contingencias a partir de las cuales se determinaran la: Sensibilidad, Especificidad, Valores predictivos (+) y (-), y Likelihood Ratio (+) y (-)

\section{Análisis de la Reproducibilidad:}

Se evaluó la confiabilidad de dos formas paralelas de obtener el diagnóstico de VDI, uno mediante el análisis visual de la coronariografía y el otro con un software cuantitativo de las imágenes coronariografícas, el estadístico utilizado fue el: Coeficiente de SpearmanBrown.

El análisis estadístico se realizó con Software estadístico SPSS versión 15.0 y se consideró un nivel de significancia del 0,05 .

\section{Resultados}

De los 54 pacientes en control post-trasplante cardíaco en el Hosp. Dr. Gustavo Fricke, en el período en que se llevó a cabo el estudio ( 3 años), se realizaron 36 coronariografías con IVUS. En 12 de ellos no se pudo realizar concomitantemente el IVUS por problemas de carácter técnico, y 6 de ellos fallecieron durante el período en estudio, por lo tanto la muestra analizada quedó conformada por 36 pacientes.

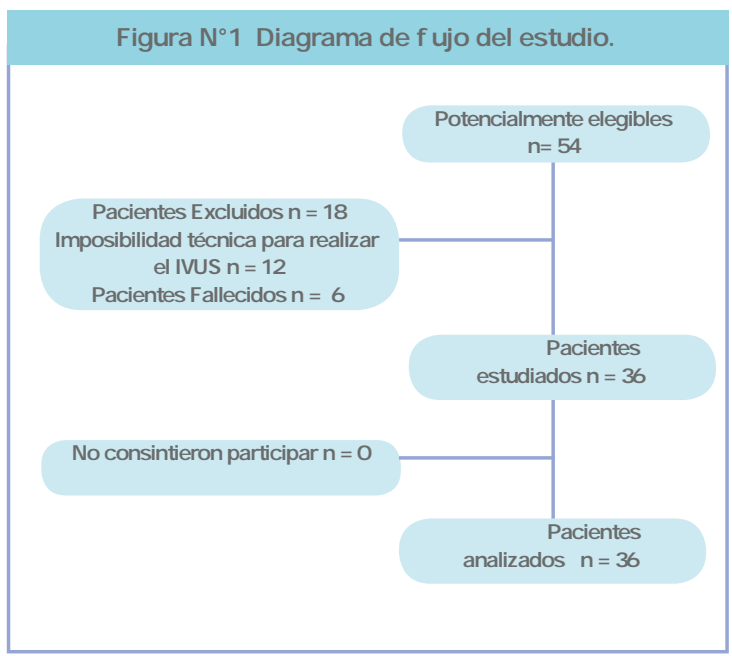

Se incluyeron 36 pacientes de los cuales, un 30,6\% estuvo compuesto por mujeres $(\mathrm{n}=11)$ y un $69,4 \%$ por hombres $(n=25)$, con una edad promedio de 44,9 \pm 12,5 años, un tiempo promedio de trasplantado de 3,7 \pm 3,7 años. El factor de riesgo más frecuente fue la Hipertensión Arterial con un 38,9\% (Ver Tabla 2).

Tabla[N $\mathrm{N}^{\circ}$ 2:[Características[de[la[muestra[en[estudio, [I] Viña[del[Mar,[Marzo[2008;[Septiembre[2010.

\section{Variable}

\begin{tabular}{|c|c|c|}
\hline \multicolumn{3}{|l|}{ Variables[demográf cas] } \\
\hline Edad_del[receptor[(años),[media[\$SD] & 0 & $44,9]+12,5$ \\
\hline Varones, $[\mathbf{n}[\%)]$ & $\square$ & $25[(69,4)$ \\
\hline \multicolumn{2}{|c|}{ 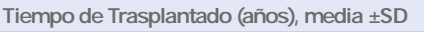 } & $3,7 \pm+3,7$ \\
\hline \multicolumn{3}{|c|}{ Factores[de[ilesgo[inmunológicos[ } \\
\hline Rechazo[agudo,[n[\%)] 四] & ? & $8(22,2)$ \\
\hline Citomegalovinus,[h[\%)] 四 & ( & $1[(2,7)$ \\
\hline \multicolumn{3}{|l|}{ Factores[de[riesgo[ho-inmunológicos] } \\
\hline Dislipidemia, $[\mathbf{n}[\%]$ & $\square$ & $11[(30,5)$ \\
\hline [Diabetes[mellitus, $[\mathbf{n}[\%)] \square]$ & $\square$ & {$[3(8,3)$} \\
\hline Tabaquismo,[h[\%)(孟血] 四 & $\square$ & $4(11,1)$ \\
\hline Hipertensión[arterial[Th(\%)四 & 0 & $14(38,9)$ \\
\hline
\end{tabular}

\section{Exactitud diagnóstica:}

Se testeo la exactitud diagnóstica de la coronariografía cualitativa y cuantitativa contra el IVUS como estándar de referencia. La tabla $\mathrm{N}^{\circ} 3$ resume estos resultados.

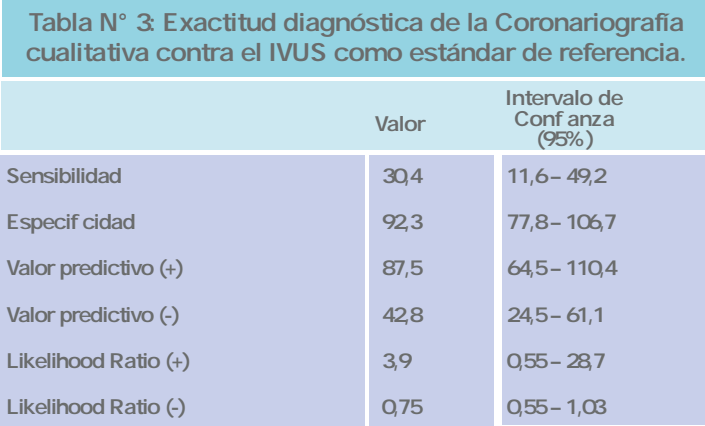

Tabla[N ${ }^{\circ}$ 4:[Exactitud[diagnóstica[de[la[Coronariografía] cuantitativa[contra[el]VUS[Como[estándar[e[eferencia.

\begin{tabular}{|c|c|c|c|c|}
\hline & & & Valor & $\begin{array}{c}\text { Intervalo de } \\
\text { Conf anza } \\
(95 \%)\end{array}$ \\
\hline Sensibilidad] & $\square$ & 口 & $52,1]$ & $31,77[72,6$ \\
\hline Especif cidad] & 口 & 口 & $69,2]$ & $44,17[94,3$ \\
\hline Valor[predictivo[]+] & ० & ૫ & 75,0 & $53,8]-[96,2$ \\
\hline Valor[predictivo[(-)] & ( & प & $45,0]$ & $23,2][66,8$ \\
\hline Likelihood[Ratio[ $[+1]$ & ( & 口 & 1,7] & $0,69-[4,2$ \\
\hline Likelihood_Ratio[-)[] & $\square$ & 口 & $0,69]$ & $0,397[1,2$ \\
\hline
\end{tabular}

\section{Reproducibilidad}

Esta fue determinada con el método de pruebas paralelas o equivalentes (split-half reliability), puesto que se está examinando la misma muestra de pacientes con 
